\title{
A hemoglobina do Pseudodoras sp., um bagre da América do Sul: isolamento, caracterizaçào e estudos dos ligantes
}

\author{
Joseph P. Martin ('); Hans J. Fyhn $\left({ }^{2}\right)$; Unni E. H. Fyhn $\left({ }^{2}\right)$; Robert L. Garlick ( $\left.{ }^{3}\right)$; Robert W. Noble ( $\left.{ }^{4}\right)$; \\ Dennis A. Powers ( $(5)$
}

\section{Resumo}

A hemoglobina do bagre da Amazônia Pseu. dodoras sp. foi isolada e caracterizada; ela compreende um componente único. A composição da subunidade da hemoglobina é similar à de outras hemoglobinas de teleósteos. O peso molecular nativo aparente determinado pela filtração em gel é 66.000. O peso molecular aparente da subunidade é 14,300 por eletroforese de diodecil sulfato de sódio. A hemoglobina não polimeriza após a oxidação por ferricianeto de potássio. A hemoglobina não apresenta efeito Root. Um pequeno efeito Bohr é evidente na hemoglobina livre de fosfato: $\Delta$ log $\mathrm{P}_{1 / 2} / \Delta \mathrm{pH}$ não é mais do que cerca de $-0.1 \mathrm{a}$ -0.2 , e aumenta para $\Delta \log \mathrm{P}_{1 / 2} / \Delta \mathrm{pH}=-0.4 \mathrm{na}$ presença de $1 \mathrm{mM}$ ATP. A cooperatividade determinada pelo $\mathbf{n}$ da equação de Hill é baixa, variando de 0.8 para 1.7 , entre os $\mathrm{pH} 6.1$ e 8.6. Os valores de $P_{1 / 2}$ das soluções de hemoglobina fracionada são extremamente baixos, menos que $0,5 \mathrm{~mm} \mathrm{Hg}$, em todos os valores de $\mathrm{pH}$ examinados entre $6,1 \mathrm{e}$ 9,0 . A alta afinidade do oxigênio é refletida principalmente na velocidade de combinação de $\mathrm{CO}$, que se assemelha às encontradas nas mioglobinas e nas subunidades isoladas da hemoglobina humana. Tanto a velocidade da combinação de $\mathrm{CO}$ quanto a velocidade da dissociação de $\mathrm{O}_{2}$ determinada por espectrofotometria de "stopped flow", são sensíveis ao $\mathrm{pH}$ e ao fosfato. Entre os $\mathrm{pH} 6,2$ e 8,1 , a velocidade de $\mathrm{CO}_{\text {on }}$ aumenta cerca de 5 vezes na hemoglobina livra de fosfato. A adição de $1 \mathrm{mM}$ ATP causa uma depressão na velocidade em todos os valores nos $\mathrm{pH}$ examinados. A velocidade de $\mathrm{O}_{2}$ off diminui 7 vezes, desde $\mathrm{pH} 6,0$ ao $\mathrm{pH} 8,2$, nas soluções de hemoglobina fracionada. A adição de $1 \mathrm{mM}$ ATP induz uma diminuição de 10 vezes através da mes- ma escala. Nos valores de $\mathrm{pH}$ abaixo de 6,0 , ocorre uma depressão na velocidade de $\mathrm{O}_{2}$ off na hemoblobina fracionada o que indica um efeito Bohr ácido.

\section{INTRODUÇÃo}

O sangue de peixe geralmente contém múltiplos componentes de hemoglobina. Bonaventura et al. (1976), por meio de eletroforese, avaliou aproximadamente 100 espécies de peixes aquáticos e marinhos. Dos peixes examinados, menos de $5 \%$ tinham hemoglobina única no sangue (Bonaventura, comunicação pessoal). Sharp (1973) estudou 31 espécies de peixe na costa da Califórnia, entre as quais descobriu só $3 \mathrm{com}$ hemoglobina única.

Riggs (1970) sugeriu que a multiplicidade da hemoglobina deve ser fisiologicamente vantajoso para os peixes. Numerosos investigadores (Hashiomoto et al., 1960; Tan et al., 1972; Gillen \& Riggs, 1973; Tan \& Noble, 1973; Weber \& DeWilde, 1975; Weber et al., 1977; Garlick et al., 1978) tem dispendido esforços consideráveis para purificar e caracterizar hemoglobinas de peixes individuais de sistemas de componentes múltiplos, com a esperança de que os papéis fisiológicos destas moléculas possam ser deduzidas. Embora as 3 hemoglobinas da carpa pareçam ser funcionalmente idênticas (Noble et al., 1970; Gillen \& Riggs, 1972), estudos cinéticos pormenorizados sobre a hemo-

( •) - Versão original inglesa publicada em Comp. Biochem Physiol. $62 \mathrm{~A}$ (1). 1979. This work was supported in part by the National Science Foundation under grant $=$ PCM $75-06451$ to the Scripps Institute of Oceonography for the supported of the Alpha Helix Program, and by the National Institutes of Health grant HLI 5460 .

(1) - Department of Zoology, Duke University Marine Laboratory, Beaufort, N.C. 28516. A predoctoral trainee supported by the National Institutes of Health grant = GMO 7184 to Duke University.

(2) - Institute of Zoophysiology, University of Oslo, Blindern, Oslo 3, Norway.

(3) - Department of Zoology, University of Texas at Austin, Austin, Texas 78712.

(4) - Department of Medicine and Biochemistry, Veterans Administration Hospital. SUNY at Buffalo, New York, 14215 Established Investigator of the American Heart Association.

(5) - Department of Biology, Johns Hopkins University Baltimore, Maryland, 21218. 
globina de espécies de peixes de componente único são comparativamente raros. Bonaventura et al., (1976) elucidaram as propriedades funcionais da hemoglobina de efeito Root do "spot" (Leiostomus xanthurus), mas nenhum outro estudo foi relatado.

As hemoglobinas de peixes podem fornecer paradigmas valiosos para o controle alostérico da afinidade ligante nas hemoglobinas dos mamíferos. Embora dois modelos padrões tenham sido propostos para esclarecer a cooperatividade da ligação de oxigênio (Monod et al., 1965), foi provado ser difícil de observarem-se estes padrões estruturais de hemoglobina humana. Entretanto, pesquisadores são capazes de experimentos com as hemoglobinas dos peixes o que foi estabilizado em altos e baixos padrões de afinidade (Noble et al., 1970; Tan et al., 1972; 1973; Tan \& Noble, 1973; Bonaventura et al., 1976).

Este trabalho descreve um estudo feito sobre a hemoglobina isolada de um bagre da Amazônia, Pseudodoras sp. $O$ sangue desta espécie contém um componente de hemoglobina. A hemoglobina do peixe foi rapidamente purificada em grande quantidade. Pseudodoras sp. é uma espécie de bagre epibêntico e experiências foram feitas para determinar se sua hemoglobina revela efeito Root. Observou-se também que as adaptações do peixe às exigências ambientais podem ser mais facilmente estudadas num sistema, em que as pressões de seleção são evidenciadas por um componente de hemoglobina único.

\section{MATERIAIS E MÉTODOS}

ISOLAMENTo - Espécies de Pseudodoras sp. foram coletadas em novembro e dezembro de 1976, durante uma expedição no R/V "Alpha Helix", no rio Amazonas, aproximadamente 50 $\mathrm{km}$ acima do rio Solimões, em sua confluência com o rio Negro. Os bagres foram apanhados com tarrafas e redes e foram transportados ao "Alpha Helix" para estudo. O sangue foi obtido por punção cardíaca e colocada em seringas heparinizadas de vidro (100 ul de heparina de sódio $(5000 \mathrm{I} . \mathrm{U} / \mathrm{ml})$ em $1,7 \%$ de $\mathrm{NaCl}$ por 5 $\mathrm{ml}$ de sangue). Foram tomadas alíquotas em tubos capilares de $50 \mu \mathrm{l}$, e centrifugadas por 3 minutos em uma centrífuga de soro, para avaliação de hematócritos. Os glóbulos vermelhos do sangue foram levados 3 vezes em 10 volumes de solução de $1 \mathrm{mM}$ Tris (refrigerados), $\mathrm{pH} 8,0,1,7 \%$ de $\mathrm{NaCl}$, e então lisadas em 3 volumes de $1 \mathrm{mM}$ Tris, $\mathrm{pH} \mathrm{8,0}$ por 1 hora, a $\mathrm{O}^{\circ} \mathrm{C}$. Foi adicionado $1 / 10$ do volume da solução de $\mathrm{NaCl}{ }_{1} \mathrm{M}$ ao hemolisado, e a mistura foi centrifugada a $28000 \mathrm{Xg}$ por 15 minutos para remover os fragmentos celulares. O sobrenadante foi então liberado de sal e fosfatos orgânicos, através de uma coluna de $2,5 \times 50 \mathrm{~cm}$ de resina de Sephadex G-25 equilibrada em 0,1 $\mathrm{mM}$ Tris, $\mathrm{pH} 8,5$. Posteriormente usou-se uma -coluna deionizadora com as seguintes resinas: $2 \mathrm{~cm}$ Dowex-50 W em forma de amônio; $2 \mathrm{~cm}$ Dowex-1 em forma de acetato, $20 \mathrm{~cm}$ Bio. Rad A 501-X8 (D) resina de "mixed bed". A hemoglobina purificada foi mantida a $5^{\circ} \mathrm{C}$ até à sua utilização. Uma amostra de eritrócitos acondicionada foi congelada a $-70^{\circ} \mathrm{C}$, transportada em gelo seco para Beaufort, N.C. e guardada a $-20^{\circ} \mathrm{C}$ por 2 meses. Em Beaufort, as células foram descongeladas, lisadas, e o peso molecular do derivado de carboxiemoglobina foi determinado pela filtração em gel.

ABREVIAÇõES - Bis tris, bis (2 hidroximetil) metano; Tris, Tris (hidroximetil) aminometano: ATP, adenosin trifosfato; EDTA, ácido etilenodiaminotetracético; I', constante da velocidade de combinação de $2^{\mathrm{a}}$ ordem para a ligação de monóxido de carbono k, constante de velocidade de dissociação de $1^{\text {a }}$ ordem; $P_{1 / 2}$, pressão parcial de oxigênio, na qual uma metade dos locais do grupo heme totais estão ocupados.

\section{ELETROFORESE}

Foi feito eletroforese em gel vertical de policrilamida ( $\mathrm{pH} 8,9,7,5 \%$ de gel) a $25^{\circ} \mathrm{C}$. segundo Davis (1964) e Ornstein (1964). Amostras de hemoglobina $(1 \mathrm{mg} / \mathrm{ml})$ no tampão da cuba superior contendo $0,1 \mathrm{M} \beta$-mercaptoetanol, e uma pequena quantidade de ditionito foram borbulhadas com monóxido de carbono e aplicadas aos geis. Albumina de soro bovino foi usada como padrão interno. Os geis foram corados por 3 horas em $0,25 \%$ de R Azul Brilhante de Coomassie em ácido acético, metanol e solução de água $(1: 2: 4)$ e descoradas por difusão. Os geis foram examinados cuida- 
dosamente a $560 \mathrm{~mm}$, usando um analisador de gel Gilford, anexado a um monocromador Beckman DU.

\section{ESTUDOS DOS PESOS MOLECULARES}

Experimentos de filtraçăo em gel das soluções de hemoglobina reduzida e oxidada dos Pseudodoras sp. foram feitos em uma coluna de $4 B$ de Sefarose $(2 X 90 \mathrm{~cm})$ em 0,05 M Tris, $\mathrm{pH} 7,5,1 \mathrm{mM}$ EDTA, a $5^{\circ} \mathrm{C}$, segundo Fyhn \& Sullivan (1975). O peso molecular da hemoglobina carboxi foi medida com uma outra resina, segundo Martin et al., (1978) .

Pesos moleculares de cadeias de hemoglobina desnaturadas foram determinadas por eletroforeses de dodecil sulfato de sódio (SDS), segundo Weber \& Osborn (1969), exceto a solução de incubação, que foi $6 \mathrm{M}$ em uréia, e $0,1 \mathrm{M}$ em $\beta$-mercaptoetanol. Transferina humana, albumina de soro bovino, ovalbumina, -quimotripsinógeno- A (pâncreàs bovino) e mioglobina de cachalote de baleia foram usados como padrões de peso molecular. A mioglobina foi obtida da Companhia Miles-Seravac. Todos os outros padrões foram obtidos da Companhia Sigma Chemical.

\section{ESTUDOS DO EQUILÍBRIO DE OXIGÊNIO}

Todos os equilíbrios de oxigênio do sangue eram feitos a $30^{\circ} \mathrm{C}$, pelo método de Powers et al., (1978), usando um analisador de dissociação de oxigênio "Hem-O-Scan" (American Instruments Corporation). Os equilíbrios de oxigênio da hemoglobina purificada foram executados a $20^{\circ} \mathrm{C}$, como descritos por Riggs \& Wolbach (1956). As soluções de hemoglobina ( $60 \mathrm{uM}$ ) foram levadas a uma intensidade força-iônica de 0,05 nos tampöes Tris e Bis-tris. Os valores de $\mathrm{P}_{1 / 2}$ foram determinados como uma função do $\mathrm{pH}$. Os experimentos foram feitos em hemolisados fracionados, na presença e na ausência de $1 \mathrm{mM}$ ATP.

\section{CINÉTICAS DE LIGANTES}

Todas as medidas cinéticas foram feitas com um aparelho de "stopped flow" do tipo originalmente descrito por Gibson \& Milnes (1964) . $\mathrm{Em}$ todos os casos, a força iônica da solução final, depois de misturada, foi de 0,05 , e quando ATP foi usado, sua concentração depois apresentadas são aos mínimos quadrantes para os primeiros $65 \%$ das reações observadas. As cinéticas da dissociação de oxigênio foram medidas pelo processo de variação do $\mathrm{pH}$, descrito por Noble et al., 1970. A hemoglobina oxigenada em $1 \mathrm{mM}$ Tris, $\mathrm{pH} 8,0$ foi misturada em uma solução de ditionito, em um tampão de força iônica de 0,1 no pH desejado. A concentração de hemoglobina final foi de aproximadamente $30 \mathrm{uM}$ em equivalentes heme, e a reação foi seguida em 540 e 560 mm.

As cinéticas da combinação de monóxido de carbono com a hemoglobina desoxigenada foram medidas misturando soluções de hemoglobina desoxigenada em tampões de força iônica de 0.1 ao pH desejado, com uma soluçăo contendo uma concentração conhecida, aproximadamente $85 \mathrm{uM}$, de monóxido de carbono dissolvido na água. Depois de misturada, a concentração de hemoglobina foi de aproximadamente $3 \mathrm{uM}$ em equivalentes heme. A reação foi seguida a 420 e $435 \mathrm{~mm}$.

\section{TAMPÕES}

Os tampões usados nas cinéticas e nos experimentos de equilíbrio foram todos de força iônica final uniforme $(I=0,05)$. Tampões Tris foram usados em experimentos acima do $\mathrm{pH} 7,0$, enquanto que os tampöes Bis-Tris foram usados em experimentos abaixo do $\mathrm{pH} 7,0$.

\section{RESULTADOS E DISCUSSÕES}

Os hematócritos dos indivíduos Pseudodoras sp. de peso 180 e 640 gramas foram $30 \%$ e $31 \%$ respectivamente. A eletroforese dos 3 hemolisados revelaram um componente de hemoglobina único.' A migração da hemoglobina nos geis de disco, em comparação com a hemoglobina humana é mostrada na fig. 1 . Depois de 3 semanas de armazenagem a $5^{\circ} \mathrm{C}$, a mobilidade do componente da hemoglobina permaneceu constante, e nenhuma heterogeneidade de carga foi detectada.

A hemoglobina não polimeriza depois do tratamento com ferricianeto de potássio, ou depois de armazenada, o que é uma característica revelada no hemolisado de Hoplias malabaricus, por Reischl (1976). Amostras de hemoglobina oxidada e reduzida elui-se como picos simétricos únicos na cromatografia 4B- Sepha- 
rose. A hemoglobina $\mathrm{CO}$ tem um peso molecular aparente de 66,000 , de acordo com a posição de eluição nos experimentos da filtração gel. A hemoglobina elui levemente, antes da hemoglobina dos "spot", que é conhecida como tetramérica $\left(\mathrm{S}_{\text {2ow }}=4.6 \mathrm{~S}\right)$, como determinada pela velocidade de centrifugação de sedimentação, sob condições de colunas específicas. O peso molecular aparente das cadeias de hemoglobina desnaturada do Pseudodoras sp. determinada pela eletroforese de gel SDS foi 14,300 , que é aproximado do obtido pelas cadelas humanas $(14,600)$.

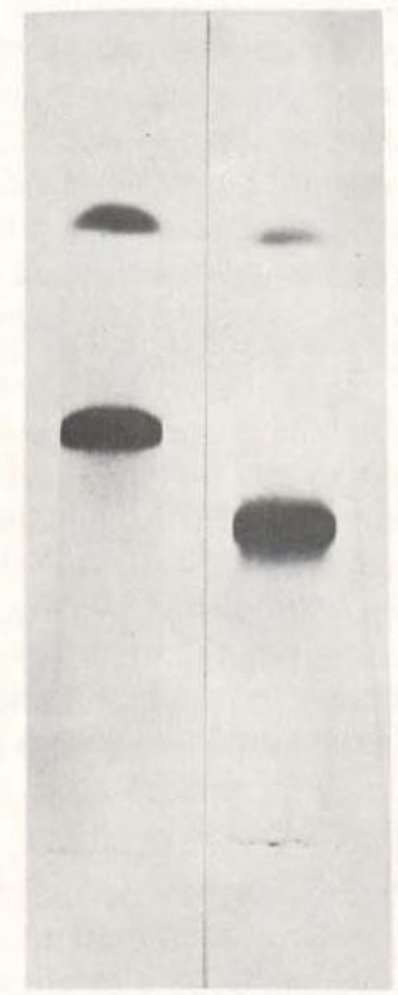

Fig. 1-Gel de disco em poliacrilamida de um hemolisado do Pseudodoras sp. (esquerda) e de um homem (direita). A banda mais anódica em ambos os geis é a albumina de soro bovino. O ânodo é o que está na parte inferior da figura.

A curva de equilíbrio de oxigênio do sangue do Pseudodoras é apresentada na fig. 2. A pH 7,6 , e a $30^{\circ} \mathrm{C}$, no glóbulo vermelho do sangue, o $P_{1 / 2}$ é $11.0 \mathrm{~mm} \mathrm{Hg}$, enquanto que sob condições de $5,6 \% \mathrm{CO}_{2}$, ela muda para 13,6 $\mathrm{mm} \mathrm{Hg}$, indiccando um pequeno efeito Bohr. O formato da curva de ligação denota uma cooperatividade do oxigênio. Os experimentos de equilíbrio do oxigênio nos hemolisados fracionados com $1 \mathrm{mM}$ ATP e sem ele (fig. 3) foram feitos a $20^{\circ} \mathrm{C}$. $O \mathrm{P}_{1 / 2}$ em $\mathrm{pH} 7,6$ da hemoglobina fracionada é $0,25 \mathrm{~mm} \mathrm{Hg}$, enquanto que a hemoglobina fracionada mais $1 \mathrm{mM}$ ATP, em pH 7,54 é de $0,56 \mathrm{~mm} \mathrm{Hg}$. O efeito Bohr dos hemolisados fracionada, $\Delta \log \mathrm{P}_{1 / 2} / \Delta \mathrm{pH}$, parece ser entre $-0,1$ e -02 . Bunn \& Riggs (1978) usando uma técnica para focalizar o ponto isoelétrico, colaboraram para este resultado. O efeito Bohr, $\Delta \log \mathrm{P}_{1 / 2} / \Delta \mathrm{pH}$ aumenta para $-0,4$ na presença de $1 \mathrm{mM}$ ATP, entre $\mathrm{pH}$ 7,0 e 9,0 , mas o valor aumenta para cerca de 1,6 na escala de $\mathrm{pH} 6,5-7,0.0 \mathrm{P}_{1 / 2}$ varia 2,5 vezes entre $\mathrm{pH} 6,5$ e 9,0 nos hemolisados fracionada, e quase 30 vezes depois das adições de ATP. Como se poderia esperar de um poliânio, o ATP exerce um grande efeito nos valores de $\mathrm{pH}$ mais baixos.

A cooperatividade entre as cadeias refletida em $n$ é geralmente baixa e é dependente do $\mathrm{pH}$ (fig. 3) . Em pH 6,1, o valor $n$ para os hemolisados fracionado é menor do que uma unidade. Entre o pH 6,7 e 8,6, $n$ obtem seu valor máximo de aproximadamente 1,7 , e depois disso declina até $\mathrm{pH} 9,0 \mathrm{n}=1,0$. A presença de $1 \mathrm{mM}$ ATP não causa aumento consistente ou diminuição no valor de $n$, acima da escala do $\mathrm{pH}$ examinado.

A velocidade de dissociação de oxigênio da hemoglobina é mostrada como uma função do $\mathrm{pH}$, com $1 \mathrm{mM}$ ATP ou sem ele, na fig. 4. A dependência do $\mathrm{pH}$ observado é a da hemoglobina com um efeito Bohr normal. $1 \mathrm{mM}$ ATP aumenta a velocidade desta reação em todos os valores de pH abaixo de 8,2 , e isto aumenta a variação total para mais de 10 vezes.

As constantes de velocidade de $2^{\mathrm{a}}$ ordem para a reação de monóxido de carbono com o derivado deoxigenado desta hemoglobina, são mostradas na fig. 5 , novamente na presença e na ausência de $1 \mathrm{mM}$ ATP. Esta constante de velocidade é fortemente dependente do $\mathrm{pH}$, aumentando enquanto o $\mathrm{pH}$ sobe de 6,0 para 8,0 , e como se poderia esperar para um efeito Bohr normal. A adição de $1 \mathrm{mM}$ ATP diminui esta velocidade de reação em todos os valores de $\mathrm{pH}$ examinados, mas o efeito é menor no $\mathrm{pH}$ mais alto. 


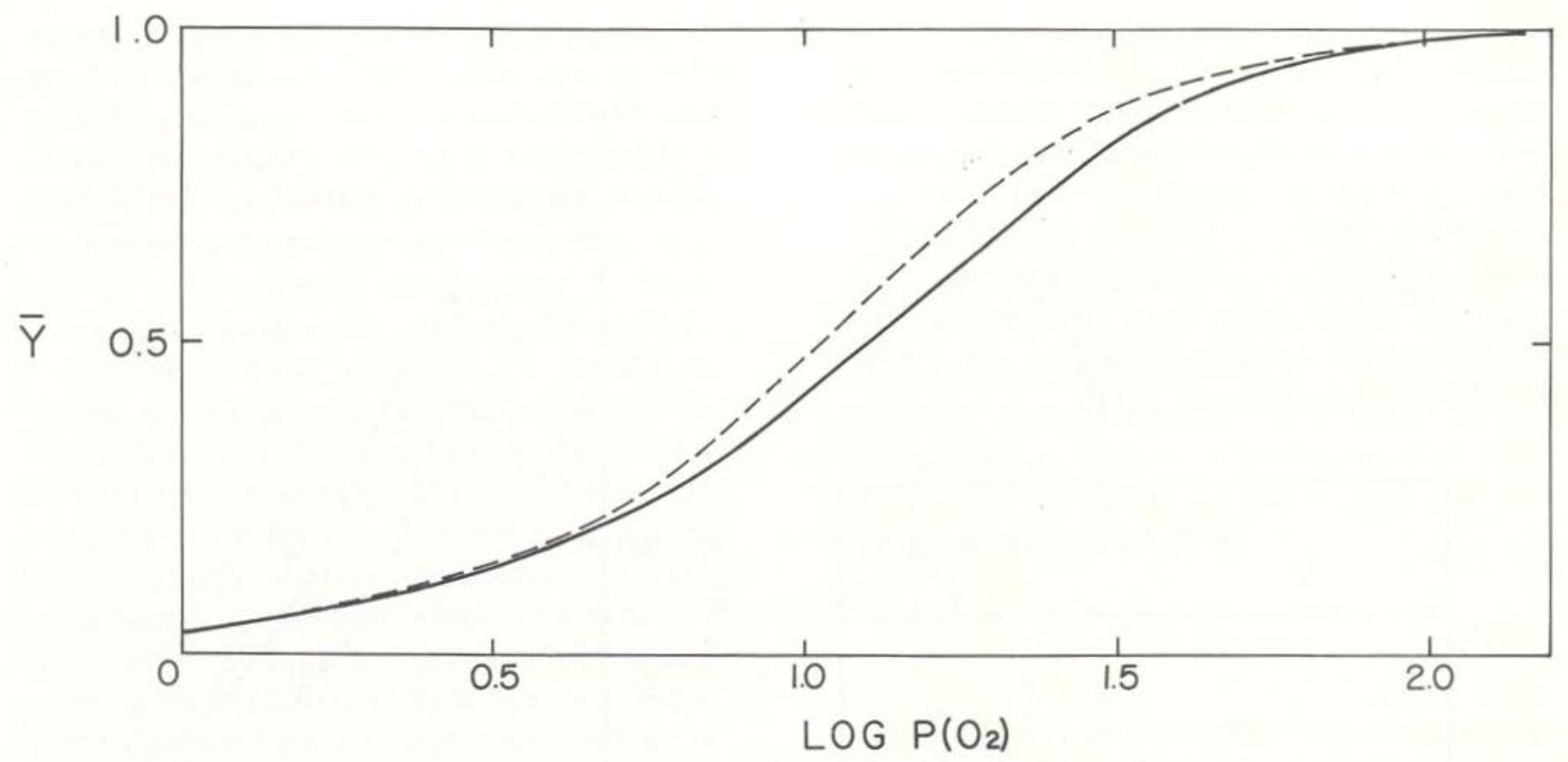

Fig. 2 - A curva de oxigenação do sangue do Pseudodoras no pH 7.6 a $30^{\circ} \mathrm{C}$. Curva 1 (-.-) e Curva 2 (-) representam a oxigenação contínua na ausência e na presença de $5.6 \%$ de $\mathrm{CO}_{2}$ respectivamente.

Embora estes resultados cinéticos indiquem um efeito Bohr normal entre $\mathrm{pH} 6,0$ e 8,0 eles sugerem um efeito Bohr ácido ou reverso, abaixo do $\mathrm{pH} 6,0$. Na ausência do ATP, isto é manifestado primariamente na velocidade de dissociaçăo de oxigênio. Na presença do ATP, são vistas, em ambas as constantes de velocidade, mas o efeito em cada uma é menor.

As constantes de velocidade para a reação com monóxido de carbono sảo as mais incomuns. Eles são muito maiores do que são as normalmente encontradas por moléculas de hemoglobina não modificadas. A velocidade observada no $\mathrm{pH} 8,0$ aproxima-se da encontrada por mioglobinas e subunidades isoladas da hemoglobina normal.

Como afirmado anteriormente, Pseudodoras $\mathrm{sp}$. difere da maioria dos peixes por possuir um único componente de hemoglobina. A simplicidade deste sistema de hemoglobina pode refletir a constância do ambiente físico e a natureza das exigências fisiológicas dos peixes. Fyhn et al., (1978) observaram que os hemolisados com uma hemoglobina única forảm mais comuns entre os peixes do alto rio Amazonas, do que entre os peixes das regiōes temperadas. A baixa variação de certos parâmetros físicos na Amazônia (isto é, a temperatura da água oscila menos que $2^{\circ} \mathrm{C}$ anualmente), e a diversidade de espécies de peixe não confrontada e a especialização do habitat na bacia Amazônica podem, em parte, explicar suas observações.

As hemoglobinas de Pseudodoras sp. foram cromatografadas como um tetrâmetro estável. Ela não se associa sob condiçöes oxidizantes, nem se dissocia em dímeros.

Em $20^{\circ}$, a hemoglobina fracionada revela um baixo $p_{1 / 2}$ para a ligação de oxigênio (menos do que $1 \mathrm{~mm} \mathrm{Hg}$ em todos os valores de $\mathrm{pH}$ examinados), e um efeito Bohr muito pequeno. A adição de $1 \mathrm{mM}$ ATP aumenta substancialmente o efeito Bohr; mas o $\mathrm{p}_{1 / 2}$ permanece relativamente baixo, nunca excedendo de 4,6 $\mathrm{mm} \mathrm{Hg}$. Em contraste, todo o sangue em $\mathrm{pH} 7,6$ e $30^{\circ} \mathrm{C}$ tem um $p_{1 / 2}$ de $11 \mathrm{~mm} \mathrm{Hg}$. O significado da diferença entre os resultados obtidos com todo o sangue, e dos resultados dos estudos da hemoglobina não podem ser avaliados sem conhecer a dependência da temperatura da afinidade de oxigênio desta hemoglobina. A diferença observada pode ser devida inteiramente às variações no parâmetro, ou pode resultar da presença, no glóbulo verme- 
Iho, de um fosfato orgânico, que é mais efetivo do que ATP. Isaacks et al. (1977) descobriram o inositol pentafosfato nos eritrócitos de outra espécie de peixe amazônico (Arapaima gigas). Tan \& Noble (1973) demonstraram com inositol hexafosfato e Torracca et al. (1977) o mostraram, para o trifosfato da guanosine, que estes são moduladores mais efetivos da ligação de oxigênio da hemoglobina do que o ATP, em algumas espécies de peixe.

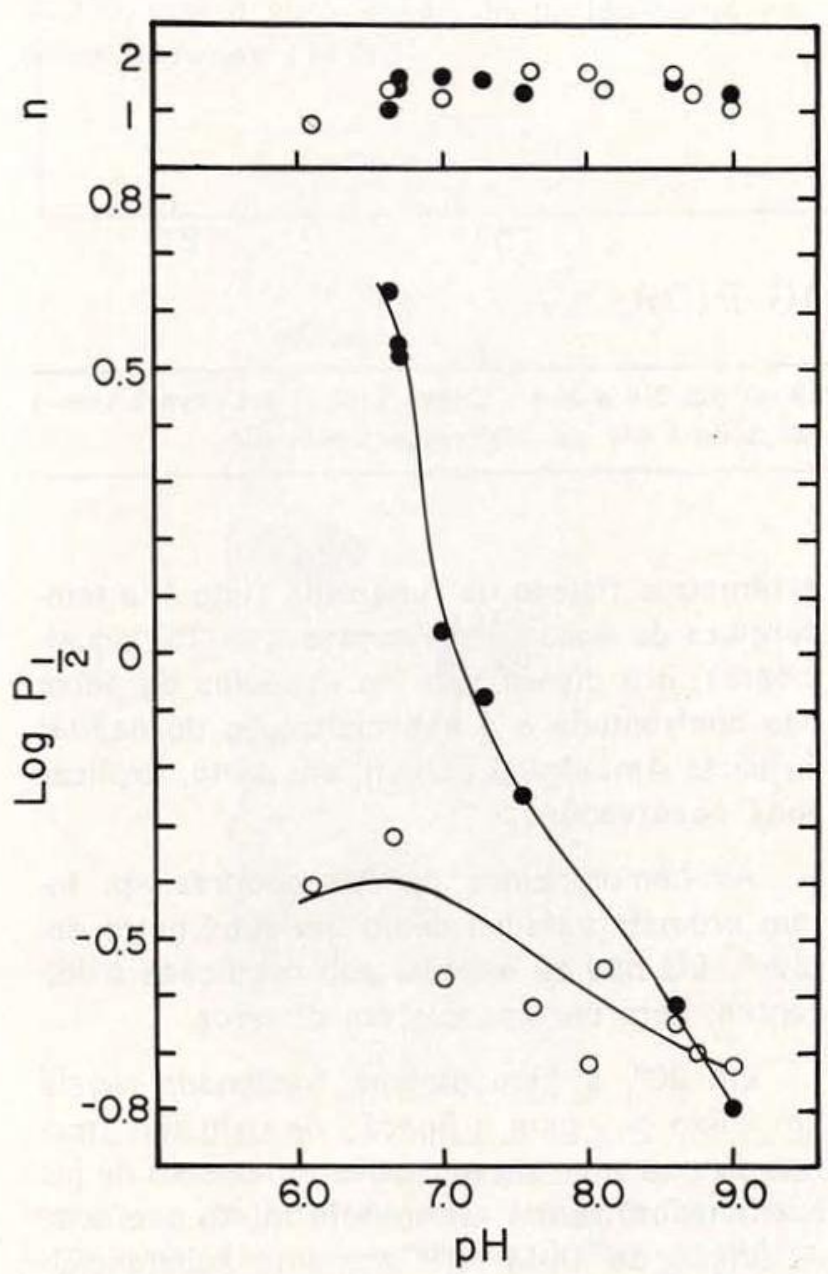

Fig. 3 - Diagramas de $\left(\log \mathrm{p}^{1 / 2}\right.$ e (n) da hemoglobina do Pseudodoras em vários valores do $\mathrm{pH}$ na condição fracionada $(O)$ e fracionada + $1 \mathrm{mM}$ ATP (•).

A constante de velocidade de $2^{\mathrm{a}}$ ordem para a associação de monóxido de carbono com a hemoglobína, colabora nos resultados de equilíbrio. Ela excede daquelas conhecidas por outras hemoglobinas de peixe por aproximada- mente uma ordem de magnitude (i. e. carpa, Noble et al., 1970; "spot", Bonaventura et al., 1976; Mylossoma sp., Martin et al., 1978) e se assemelha aos valores obtidos por subunidades de hemoglobina isoladas e mioglobinas.

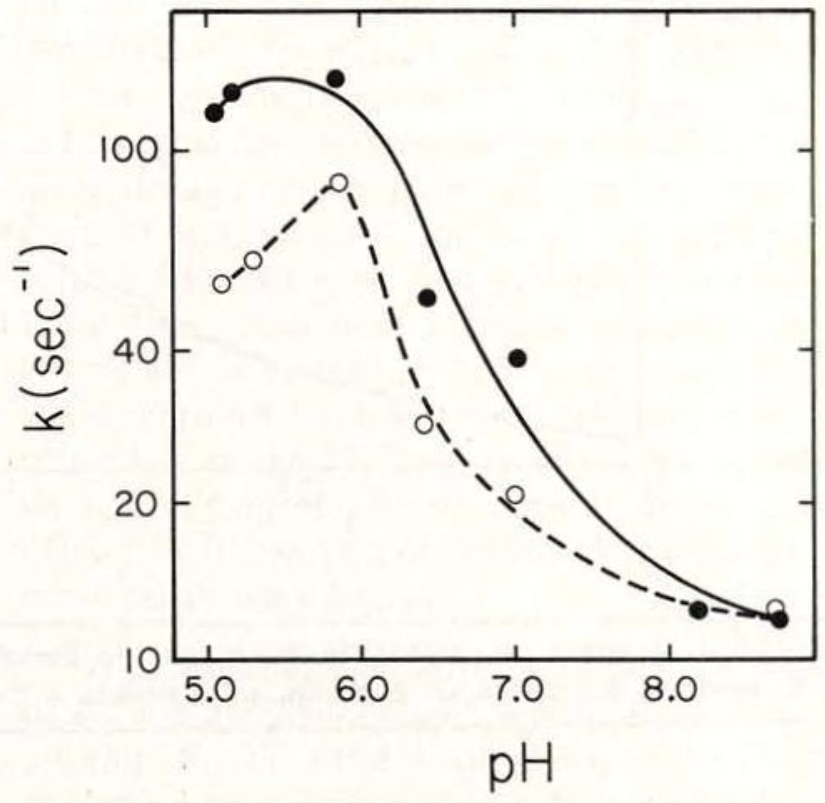

Fig. 4-A relação entre $\mathrm{o} \mathrm{pH} \mathrm{e}$ a constante de dissociação de $10^{\circ}$ ordem (k) da oxiemoglobina do Pseudodoras. As condições são descritas no texto. O hemolisado fracionado $+1 \mathrm{mM}$ ATP $(\bullet)$.

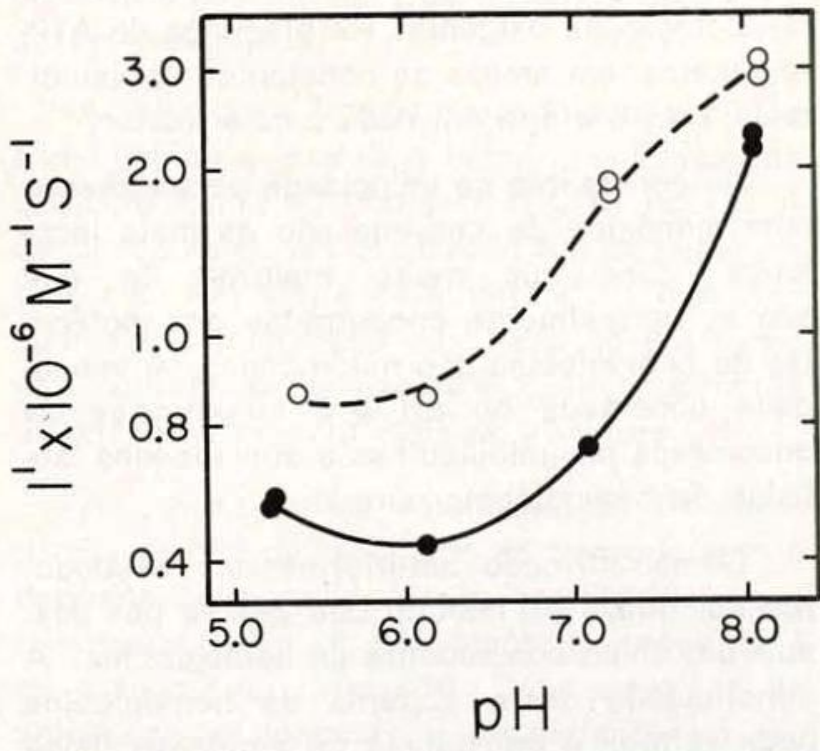

Fig. 5 - A relação entre o pH e a constante de velocidade de combinação de $2 .^{\circ}$ ordem da hemoglobina do Pseudodoras para o monóxido de carbono (1'). As condições estäo descritas no texto. O hemolisado fracionado $(O)$, hemolisado "stripped" $+1 \mathrm{mM}$ ATP (•). 
Outras características notáveis da hemoglobina do Pseudodoras são seu pequeno efeito Bohr, e a ausência de um efeito Root. Riggs (1970) apontou que a magnitude do efeito Bohr na hemoglobina dos peixes é freqüentemente proporcional ao nível de atividades dos mesmos. Os dados sobre a hemoglobina do Pseudodoras são consistentes, com a generalização de que Pseudodoras é um bagre epibêntico $\boldsymbol{\theta}$, como um siluriforme, é geralmente menos ativo do que peixe de outra velocidade. Conseqüentemente, um grande efeito Bohr pode não ser exigido para facilitar a descarga de oxigênio aos tecidos. Embora Pseudodoras possua bexiga natatória, não tem comumente presente

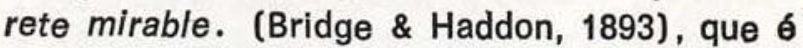
o sistema multiplicador contracorrente, no qual a hemoglobina libera o oxigênio para entrar na bexiga natatória. Em adição, Pseudodoras não tem um rete coróide (Farmer et al., 1978), a estrutura responsável pela entrega de oxigênio para o olho. A ausência destas trocas anatômicas impediram uma hemoglobina com efeito Root.

O ATP e $\mathrm{H}+$ afetam tanto as velocidades $\mathrm{CO}_{\text {on }}$ quanto a de $\mathrm{O}_{2 \text { off }}$ da hemoglobina dos Pseudodoras. Isto é similar aos efeitos destes agentes em outras hemoglobinas de peixes. A magnitude destas mudanças cinéticas entre $\mathrm{pH} 5,1$ e 8,7 , esclarece adequadamente a mudança de aproximadamente 30 vezes no $p_{1 / 2}$ dos hemolisados fracionados na presença de $1 \mathrm{mM}$ ATP. Os dados de equilíbrio sugerem um efeito Bohr ácido num $\mathrm{pH}$ baixo, sımilar ao observado na hemoglobina da truta 3 (Salmo galrdneri) (Lau et al., 1975) . O comportamento das constantes $\mathrm{CO}_{\text {on }}$ e $\mathrm{O}_{\text {off, abaixo do }} \mathrm{pH}$ 6,0 na hemoglobina do Pseudodoras sustenta os dados de equilíbrio. Entretanto, o pH no qual o efeito Bohr aparece primeiramente, é mais alto no equilíbrio do que nos experimentos cinéticos, isto possivelmente como uma consequêencia das diferenças no comportamento de ligantes do $\mathrm{CO}$ e $\mathrm{O}_{2}$.

Os valores $n$ obtidos da análise dos dados de equilíbrio, variam como uma função do $\mathrm{pH}$. A cooperatividade máxima é adquirida na extensẫo do $\mathrm{pH}$ fisiológico, como na hemoglobina da carpa (Noble et al., 1970); $\boldsymbol{n}$ diminui acima do $\mathrm{pH} 8,6$ e abaixo do $\mathrm{pH} 6,7$. O ATP não al- tera significativamente este padrão. No $\mathrm{pH}$ 6,1 , a cooperatividade cai abaixo da unidade, indicando talvez as diferenças das afinidades ligantes entre as cadeias $\propto$ e $\beta$.

Concluindo, a hemoglobina do Pseudodoras compartilha muitas das características dos IIgantes das hemoglobinas do efeito Root (por exemplo as da carpa, truta e "spot") embora ela não tenha um efeito Root. Comparações estruturais pormenorizadas entre estas hemoglobinas podem, todavia, elucidar-nos com respeito à base estrutural do efeito Root nas hemoglobinas de peixe.

\section{AgRADECIMENTOS}

Gostaríamos de agradecer aos oficiais e à tripulação do "Alpha Helix", por sua cooperação e camaradagem durante este cruzeiro. Gostaríamos de expressar também nossos agradecimentos ao Governo brasileiro por permitir-nos trabalhar nas águas do rio Amazonas. Hans J. Fyhn e Unni E. H. Fyhn agradecem o auxílio do "Norwegian Research Council for Science and the Humanities" (Conselho Norueguês de Pesquisas para Ciências e Humanidades). A ajuda adicional fol dada por NSF DEB 76-19877 (para D. A. Powets), a Sociedade Geográfica Nacional (The National Geographic Society) (para D. A. Powers); ajuda NSF-PCM-06719, e auxílio NIH-GM 21314 (para A. Riggs) .

\section{SUMMARY}

The hemoglobin of the Amazonian Catfish Pseudodoras sp. was isolated and characterized; it comprises a single component. The hemoglobin's subunit composition is similar to that of other teleost hemoglobins. The apparent native molecular weight as determined by gel filtration is 66,000 . The apparent subunit molecular weight is 14,300 by sodium dodecyl sulfate electrophoresis. The hemoglobin does not polymerize after oxidation by potassium ferricyanide. The hemoglobin lacks a Root effect. A small Bohr effects is evident in the phosphate free hemoglobin: $\Delta \log \mathrm{p}^{1 / 2} / \Delta \mathrm{pH}$ is no more than about -0.1 to -0.2 and increases to $\Delta \log 1 / 2 / \Delta \mathrm{pH}=-0.4$ in the presence of $1 \mathrm{mM}$ ATP. The cooperativity, as determined by $n$ of the Hill equation, is low, varying from 0.8 to 1.7 
between $\mathrm{pH} 6.1$ and 8.6 . The $\mathrm{p}_{1 / 2}$ values of stripped hemoglobin solutions are extremely low, less than $0.5 \mathrm{~mm} \mathrm{Hg}$ at all $\mathrm{pH}$ values examined between $\mathrm{pH}$ $6.1^{\circ}$ and 9.0 . The high oxygen affinity is reflected primarily in the $\mathrm{CO}$ combination rate with resembles that found in myoglobins and isolated subunits of human hemoglobin. Both the $\mathrm{CO}$ combination rate and the $\mathrm{O}_{2}$ dissociation rate determined by stopped flow spectrophotometry are $\mathrm{pH}$ and phosphate sensitive. Between $\mathrm{pH} 6.2$ and 8.1 the $\mathrm{CO}_{\mathrm{on}}$ rate increases about 5-fold in the phosphate-free hemoglobin. Addition of $1 \mathrm{mM}$ ATP causes a depression in the rate at all $\mathrm{pH}$ values examined. The $0_{2 \text { off }}$ rate decreases 7-fold going from pH 6.0 to 8.2 is stripped hemoglobin solutions. Addition of $1 \mathrm{mM}$ ATP inducesa ten-fold decrease over the same range. At $\mathrm{pH}$ values below 6.0 a depression in the $0_{2 \text { off }}$ rate occurs in the stripped hemoglobin, indicative of an acid Bohr effect.

\section{BIBLIOGRAFIA}

Bonaventura, C.; Sullivan, B.; Bonaventura, J. \& BRUNORI, M.

1976 - Spot hemoglobin, studies on the root effect hemoglobin of a marine teleost. J. Biol. Chem., 251:1871-1876.

BRIDGE, S.W. \& HADDON, G.C.

1893 - Contributions to the anatomy of fishes. II. The air bladder and Weberian ossicles in the siluroid fishes. Phil. Trans. (B), $184: 65-434$.

BRUNORI, M.

1975 - Molecular adaptation to physiological requerements: The hemoglobin systems of trout. Curr. Tropics in. Cell. Regulation 9, 1-39 (Horecker, $M$. and Stadtman, E., eds. Academic Press, New York.

BRUNORI, M.; FALCIONE, G.; Fortuna, G. and GIARDINA, B.

1975 - The effects of anions on the oxygen binding properties of the hemoglobin components from trout, (Salmo Irideus) Arch. Biochem, Biophys. $168: 512-519$.

BUNN, H.F. \& RIGGS, A.

1978 - A medida do efeito Bohr em hemoglobinas de peixe por focalização elétrica em gel. Acta Amazonica 8(4): Suplemento. (Este volume).

DAvis, B.J.

1964 - Disc electropheresis - II. Method and application to human serum proteins. Ann. N. Y. Acad. Sci., $121: 404-427$.
FARMER, M.; FYHN, H.J.; FYHN, U.E.H. and NOBLE, R.M.

1978 - Ocorrência de hemoglobinas de efeito Root em peixes amazônicos. Acta Ama. zonica $8(4)$ : Suplemento. (Este volume)

FYHN, U.E.H.; FYhN, H.J.; DıvIs, B.J.; FINK, W.L.; Powers, D.A. \& Garlick, R.L.

1978 - Heterogeneidade de hemoglobina nos peixes da Amazônia. Acta Amazonica 8(4) : Suplemento. (Este volume).

FYhN, U.E.H.; and SUlLIVAN, B.

1975 - Elasmobranch hemoglobins: Dimerization and polymerization in various species. Comp. Biochem. Physiol. 50B, 119-129.

GNRLICK, R.L.; BUNN, H.F.; FYHN, H.J.; FYhN, U.E.H.; Martin, J.P.; Noble, R.W. \& POWERS, D.A.

1978 - Estudos funcionais na hemoglobina de componente simples do peixe faca amazônico, Sternopygos macrurus. Acta Amazonica 8(4): Suplemento. (Este volume).

GIBSON, Q.H. \& MILNES, L.

1964 - Apparatus for rapid and sensitive spectrophotemetry Biochem, J., 91 : 161-171.

GILLEN, R. \& RIGGS, A.

1972 - Structure and function of the hemoglobins of the carp, Cyprinus carpio. J. Biol. Chem., 247 : 6039-6046.

1973 - Structure and function of the isolated hemoglobin of the American eel Anguilla rostrata. J. Biol. Chem., 248:1961-1969.

Hashimoto, K.Y. Yamaguchi \& MatsuURa, F.

1960 - Comparative studies on two hemoglobins of Salmon - IV: Oxigen dissociation curves. Bull. Jap. Soc. Sci. Fish, $26: 827-834$.

ISAMCKS, R.E.; KIM, H.D.; BARTLETT, G.R. \& HARKNESS, D.R.

1977 - Inosotol pentaphosphate in erythrocytes of a freswater fish, piraracu (Arapaima gigas) Life Sciences 20:987 990 .

Lau, H.K.F.; Wallach, D.; Pennelly, R. \& Noble, R.W.

1975 - Ligand binding properties of hemoglobin 3 of the thout, Salmo gairdneri. J. Biol. Chem., 250:1400-1404.

Martin, J.P.; Bonaventura, J.; Brunori, M.; Fyhn, H.J.; FYhN, U.E.H.; Garlick, R.L.; POWERS, D.A. \& WIISON, M.T.

1978 - Isolamento e caracterização dos componentes da hemoglobina de Mylossoma sp., um teleósteo da Amazônia, Acta Amazonica 8(4): Suplemento. (Este volume). 
Monod, J.; Wyman, J. \& Changenus, J.P.

1965 - On the nature of allosteric transitions: A plausible model. J. Molec. Biol., 12 : 88-118.

Noble, R.W.; Parhurst, L.J. \& Gibson, Q.H.

$1970-$ The effect of $\mathrm{pH}$ on the reactions of oxygen and Carbon monoxide with the hemoglobin of carp. Cyprinus carpio. J. Biol. Chem., 245 : 6628-6633.

ORNSTEIN, L.

1964 - Disc electrophoressis - I. Background and theory. Ann. N. Y. Acad. Sci., 121 : 321-349.

POWERS, D.A.; FYhN, H.J.; FYhN, U.E.H.; MARTIN, J.P.; GARLICK, R.L. \& WoOd, S.C.

1978 - Estudo comparativo do equilíbrio de oxigênio no sangue de 40 gêneros de peixes da Amazônia. Acta Amazonica 8(4) : Suplemento. (Este volume).

REISHL, E.

1976 - The hemoglobin of a fresh water teleost Hoplias malabaricus: Heterogeneeity and polymerization. Comp. Biochem. Physiol., 55B : 255-247.

Rigos, A.

1970 - Properties of fish hemoglobins. In: Fish Physiology (HOAR, W.S. \& RANDALL, D.J., eds). New York. Academic Press, Vol. IV. pp. 209-251.

Riggs, A. \& Wolbach, R.A.

1956 - Sulfhudryl groups and the structure of hemoglobin. J. Gen. Physiol. 39 : 585-605.

SHARP, G.D.

1973 - An electrophoretic study of hemoglobins of some scombrid fishes and related forms. Comp. Biochem. Physiol, 44B : 381-388.

TAN, A.; DeYoung, A. \& Noble, R.W.

1972 - The $\mathrm{pH}$ dependence of the affinity, kinetics, and cooperativity of ligand binding to carp hemoglobin Cyprinus carpio. J. Biol. Chem., 247 : 2493-2498.
TAN, A.L. \& Noble, R.W.

1973 - The effect of inositol hexaphosphate on the allosteric properties of carp hemoglobin. J. Biol. Chem., 248 : 7412-7416.

TAN, A.; Noble, R.W. \& Gibson, Q.H.

1973 - Conditions restricting allosteric transition in carp hemoglobin. J. Biol. Chem., $248: 2880-2888$.

TORRACCA, A.M.; RASChETTI, R.; SALVIOLA, R.; RiccionRdi, G. \& Winterhalter, K.H.

1977 - Modulation of the Root effect in goldfish by ATP and GTP. Biochem. Biophys. Acta., 496 : 367-373.

WeBer, R.E. \& OSBORN, M.

1969 - The reliability of molecular weight determinations by dodecyl sulfatepolyactylamide electrophoresis. J. Biol. Chem., 244 : 440-4412.

WeBER, R.E. \& DEWILDE, J.A.M.

1975 - Oxygenation properties of hemoglobin from the flafish plaice (Pleuronectes platissa) and flouder (Platicthys flesus) J. Comp. Physiol., 101 : 99-110.

Weber, R.E.; Johansen, K.; LyKKeboe, G. \& Maloiy, G.M.O.

1977 - The oxigen binding properties of hemoglobins estivating and actibe African lungfish. J. Exp. Zool., $199:$ 85-99.

Weber, R. E.; Sullivan, B.; Bonaventura, J. \& BONAVENTURA, C.

1976 - The hemoglobin of the primitive fish, Amia calva: Isolation and functional characterization of the individual hemoglobin components. Biochim. Biophys. Acta., $434:$ 18-31.

YАMAgUCh, K.; КосніуамА, Y.; НАSнimoto, K. \& MATSUURA, F.

1962 - Studies on the multiple hemoglobins of eel II. Oxygen dissociation curve and the relative amounts of components. F. \& S. Bull. Jap. Soc. Sci. Fish., 28 : $192-200$.

YAMAgUch, K. \& MatsuURA, F.

1963 - Studies on two hemoglobins of Loach II. Oxygen dissociation curve. Bull. Jap. Soc. Sci. Fish., $29: 180$. 